\title{
Correction to: Understanding Well-Being in the Ghanaian Context: Linkages between Lay Conceptions of Well-Being and Measures of Hedonic and Eudaimonic Well-Being
}

\author{
Angelina Wilson Fadiji ${ }^{1,2} \cdot$ Leana Meiring $^{3} \cdot$ Marie P. Wissing ${ }^{2}$ \\ Published online: 6 February 2020 \\ (C) The International Society for Quality-of-Life Studies (ISQOLS) and Springer Nature B.V. 2020
}

\section{Correction to: Applied Research in Quality of Life https://doi.org/10.1007/s11482-019-09777-2}

The authors would like to correct an error in Table 2 of the published article. This table presents the model fit indices for each of the models tested for the SWLS and the MLQ. In the table, we indicated that the CFI fit indices for MLQ model 3 was .052. This figure is wrong as it demonstrates a poor fit index, whereas the correct value for MLQ model is supposed to be .921 showing an acceptable model fit based on the CFI value.

Shown below is Table 2 with the correct values.

Table 2 Modification Indices for SWLS and MLQ

\begin{tabular}{llllr}
\hline Modification indices & RMSEA & SRMR & CFI & TLI \\
\hline Model 1: SWLS & $.075[90 \%$ CI: .037, .116] & .030 & .969 & .939 \\
Model 2: MLQ with item 9 & $.106[90 \%$ CI .092,.121] & .079 & .842 & .791 \\
Model 3. MLQ without item 9 & $.079[90 \%$ CI: $.062, .097$ & .052 & .921 & .891 \\
\hline
\end{tabular}

Note: Satisfaction with life (SWLS); Meaning in Life -Presence (MLQ-P); Meaning in Life -Search (MLQ-S)

The online version of the original article can be found at https://doi.org/10.1007/s11482-019-09777-2

Angelina Wilson Fadiji

Wilson.angelina1311@gmail.com

1 Education and Skills Development Unit, Human Sciences Research Council, Cape Town, South Africa

2 Africa Unit for Transdisciplinary Health Research, Faculty of Health Sciences, North West University, Potchefstroom Campus, Potchefstroom, South Africa

3 Reviews and Evaluation, National Research Foundation, Pretoria, South Africa 